\title{
An interaction of heart disease-associated proteins POPDC1/2 with XIRP1 in transverse tubules and intercalated discs
}

\author{
Ian Holt ${ }^{1,2}$, Heidi R. Fuller ${ }^{1,2}$, Roland F. R. Schindler ${ }^{3}$, Sally L. Shirran ${ }^{4}$, Thomas Brand ${ }^{3}$ and Glenn E. Morris ${ }^{1,2}$
}

\begin{abstract}
Background: Popeye domain-containing proteins 1 and 2 (POPDC1 and POPDC2) are transmembrane proteins involved in cyclic AMP-mediated signalling processes and are required for normal cardiac pacemaking and conduction. In order to identify novel protein interaction partners, POPDC1 and 2 proteins were attached to beads and compared by proteomic analysis with control beads in the pull-down of proteins from cultured human skeletal myotubes.
\end{abstract}

Results: There were highly-significant interactions of both POPDC1 and POPDC2 with XIRP1 (Xin actin binding repeatcontaining protein 1), actin and, to a lesser degree, annexin A5. In adult human skeletal muscle, both XIRP1 and POPDC1/2 were present at the sarcolemma and in T-tubules. The interaction of POPDC1 with XIRP1 was confirmed in adult rat heart extracts. Using new monoclonal antibodies specific for POPDC1 and POPDC2, both proteins, together with XIRP1, were found mainly at intercalated discs but also at T-tubules in adult rat and human heart.

Conclusions: Mutations in human POPDC1, POPDC2 and in human XIRP1, all cause pathological cardiac arrhythmias, suggesting a possible role for POPDC1/2 and XIRP1 interaction in normal cardiac conduction.

Keywords: Popeye domain-containing, Xin actin binding repeat-containing, Cardiac conduction, Intercalated discs, Transverse tubules

\section{Background}

Popeye domain containing protein 1 (POPDC1), also known as blood vessel epicardial substance (BVES), was first found in chicken heart by subtractive hybridisation $[1,2]$. Popdc1 and two related gene family members, Popdc2 and Popdc3, were identified in mammals and shown to be developmentally regulated and preferentially expressed in cardiac and skeletal muscle [2]. Human $P O P D C 1$ is found on chromosome $6 \mathrm{q} 21$ along with $P O P D C 3$ in tandem array, whereas POPDC2 is found on human chromosome 3q13.33. The POPDC proteins are

\footnotetext{
* Correspondence: ian.holt@nhs.net

'Wolfson Centre for Inherited Neuromuscular Disease, RJAH Orthopaedic Hospital, Oswestry SY10 7AG, UK

${ }^{2}$ School of Pharmacy and Bioengineering, Keele University, Keele ST5 5BG, UK Full list of author information is available at the end of the article
}

highly conserved throughout the animal kingdom, suggesting that they play an essential role [3].

POPDC proteins consist of a short extracellular Nterminal sequence which is glycosylated, three transmembrane domains, a conserved intracellular Popeye domain and a variable $\mathrm{C}$-terminal domain which is isoform-specific, contains regions of low complexity and may be phosphorylated [4]. POPDC1 exists at the plasma membrane as a homodimer, which is stabilised by disulphide bonds [5, 6]. The predicted secondary structure of the Popeye domain contains a cyclic nucleotide binding domain, which binds the second messenger cyclic adenosine $3^{\prime}, 5^{\prime}$-monophosphate (cAMP) with high affinity [7]. Interaction between POPDC proteins and the potassium two pore domain channel subfamily $\mathrm{K}$ member 2 (KCNK2, also known as TREK-1) has been demonstrated, which leads to an increase in KCNK2

(c) The Author(s). 2020 Open Access This article is licensed under a Creative Commons Attribution 4.0 International License, which permits use, sharing, adaptation, distribution and reproduction in any medium or format, as long as you give appropriate credit to the original author(s) and the source, provide a link to the Creative Commons licence, and indicate if changes were made. The images or other third party material in this article are included in the article's Creative Commons licence, unless indicated otherwise in a credit line to the material. If material is not included in the article's Creative Commons licence and your intended use is not permitted by statutory regulation or exceeds the permitted use, you will need to obtain permission directly from the copyright holder. To view a copy of this licence, visit http://creativecommons.org/licenses/by/4.0/ The Creative Commons Public Domain Dedication waiver (http://creativecommons.org/publicdomain/zero/1.0/) applies to the data made available in this article, unless otherwise stated in a credit line to the data. 
current in isolated mouse sinus node myocytes, and this activity was reduced by an increase in cAMP levels [7]. A number of other membrane proteins have been reported to interact with POPDC proteins, including caveolin-3 (CAV3) in mouse cardiomyocytes, which is a major component of caveolae in striated muscle membranes [8].

A homozygous missense variant in POPDC1 has been found in a family with cardiac arrhythmia and limbgirdle muscular dystrophy (LGMD). This autosomal recessive mutation in POPDC1 is associated with reduced cAMP affinity [9]. More recently, three homozygous loss-of-function mutations in $P O P D C 1$ were identified in three families with LGMD and cardiac conduction abnormalities [10] and a missense mutation in POPDC1 was observed in a patient with contractures and possible mild cardiac involvement [11]. A heterozygous nucleotide substitution in $P O P D C 2$ has been associated with severe atrioventricular block [12] and homozygous missense variants in POPDC3 have been associated with limb girdle muscular dystrophy in the absence of a cardiac phenotype [13]. POPDC1 protein was down-regulated with abnormal immunolocalisation in failing human hearts and POPDC1 and POPDC3 mRNA levels were reduced in the left ventricles of end-stage failing hearts [14]. Popdc1 null mice showed impaired skeletal muscle regeneration [15] and increased sensitivity towards ischemia reperfusion [8]. Moreover, mice with null-mutations in Popdc1 or Popdc2 developed a stress-induced sinus node bradycardia due to pacemaker dysfunction $[7,16]$. Knockdown of popdc2 in zebrafish by injecting embryos with morpholino oligonucleotides resulted in the aberrant development of skeletal muscle and heart. A reduction in oligonucleotide concentration lead to an improvement in the skeletal muscle pathology, but abnormalities in the cardiac conduction system remained, resulting in cardiac arrhythmia and a reduction in heart rate [17].

Immunolocalization studies with polyclonal antibodies have shown that POPDC1 and POPDC2 generally localise to the sarcolemma of control skeletal muscle, but this membrane localisation was greatly reduced in muscle tissue from patients with pathogenic mutations in POPDC1 $[9,10]$. In the heart, POPDC1 and POPDC2 were found at the plasma membrane of cardiomyocytes, with high levels in the cardiac conduction system $[7,18]$.

In addition to the essential roles that POPDC proteins play in the maintenance of structure and function of skeletal muscles and in cardiac pacemaking and conduction, POPDC1 may play a role in tumor formation [19]. POPDC1 is thought to have a tumor suppressor function and decreased POPDC1 expression due to DNA methylation occurs in the early stages of a number of cancers [reviewed: [20, 21]].

Here we report a proteomic study to identify novel POPDC1/2-interacting proteins. The most significant hit with both POPDC1 and POPDC2 was XIRP1 (Xin actin binding repeat containing 1 , also known as Xin or cardiomyopathy-associated protein 1 (CMYA1)). Pull-down and co-immunoprecipitation experiments confirmed the interaction of XIRP1 with POPDC1 and POPDC2. POPDC1/2 and XIRP1 displayed colocalization at the intercalated disk and in T-tubules. Mutations for both genes in model organisms $[7,17,22,23]$ and patients $[9,10,24]$ have been associated with severe cardiac conduction defects and impaired skeletal muscle regeneration was reported for both Popdc1 [15] and Xirp1 [25, 26] null mutants. This suggests some overlapping function between POPDC1 and XIRP1, which could be based in part on the interaction reported here.

\section{Results}

XIRP1 and cardiac actin are the highest-scoring binding partners for both POPDC1 and POPDC2 in skeletal muscle myotubes

With the goal of identifying novel protein interaction partners for POPDC proteins in skeletal muscle, POPDC proteins available as GST-fusion "bait" proteins (mouse POPDC1 or POPDC2) were attached to glutathione beads and used to pull-down proteins from RIPA extracts of human skeletal myotubes. Proteins pulled down by the beads were identified by mass spectrometry (unfiltered results shown in: Additional file 1). Cardiac actin and XIRP1 had the highest scores for a number of significant peptides identified by mass spectrometry (Table 1 ) for both mouse POPDC1 and mouse POPDC2, after eliminating nonspecific binding of proteins to control beads (no fusion protein attached). Annexin A5 was also present in both pull-downs. For further study, we selected from Table 1 those proteins with known involvement in cardiac conduction and / or cardiomyopathy: XIRP1 and annexin A5.

Cardiac actin is the principal actin isoform during early stages of skeletal myogenesis and is only gradually replaced by skeletal muscle actin in adult skeletal muscle [27], so it is not surprising that the cardiac isoform was detected in myotube extracts.

\section{Confirmation by western blotting of POPDC1 interaction with XIRP1 in extracts of both human myotube cultures and adult rat heart}

The presence of XIRP1 in POPDC1/2 pull downs was confirmed by western blotting with a commercial antiXIRP1 antibody (Fig. 1a upper blot). Figure 1a lower blot is a loading control showing antibody against the GST "bait" protein. XIRP1 was also immunoprecipitated from the same human myotube extracts by mAb $1 \mathrm{C} 12$ against POPDC1 (Fig. 1b). Western blots are representative of pull-downs repeated using three separate extracts of the cultured myotube cell line. Recombinant POPDC1 attached to glutathione beads also pulled-down XIRP1 from a RIPA extract of adult rat heart ventricle (Fig. 1d). 
Table 1 Mass spectrometry abridged results of mouse POPDC1/2 pull-downs

\begin{tabular}{|c|c|c|c|}
\hline & Accession number & Number of & ptides \\
\hline & & mPOPDC1 & mPOPDC2 \\
\hline Actin, alpha cardiac muscle 1 & ACTC_HUMAN & 12 & 12 \\
\hline Xin actin-binding repeat-containing protein 1 & XIRP1_HUMAN & 11 & 19 \\
\hline Tubulin beta-4B chain & TBB4B_HUMAN & 9 & 7 \\
\hline Tubulin beta chain & TBB5_HUMAN & 9 & 6 \\
\hline Tubulin beta- 6 chain & TBB6_HUMAN & 9 & 4 \\
\hline ATP synthase subunit beta, mitochondrial & ATPB_HUMAN & 7 & 5 \\
\hline Fructose-bisphosphate aldolase A & ALDOA_HUMAN & 5 & 7 \\
\hline L-lactate dehydrogenase A chain & LDHA_HUMAN & 5 & 5 \\
\hline Histone H2AX & H2AX_HUMAN & 4 & 4 \\
\hline PDZ and LIM domain protein 3 & PDLI3_HUMAN & 3 & 5 \\
\hline Glucosidase 2 subunit beta & GLU2B_HUMAN & 3 & 5 \\
\hline Lamina-associated polypeptide 2 (beta/gamma) & LAP2B_HUMAN & 3 & 4 \\
\hline Histone H2A.V & H2AV_HUMAN & 3 & 4 \\
\hline Phosphoglycerate mutase 1 & PGAM1_HUMAN & 3 & 4 \\
\hline Phosphoglycerate kinase 1 & PGK1_HUMAN & 3 & 4 \\
\hline Heat shock protein beta-2 & HSPB2_HUMAN & 3 & 4 \\
\hline Annexin A5 & ANXA5_HUMAN & 3 & 3 \\
\hline $40 \mathrm{~S}$ ribosomal protein $\mathrm{S} 30$ & RS30_HUMAN & 3 & 3 \\
\hline
\end{tabular}

Only proteins that met the following criteria are shown: identified from more than two significant unique peptide sequences, undetected in the control pull-down AND detected across both mPOPDC1 and mPOPDC2 pull-downs. See Additional file 1 for full results

The pull down of annexin A5 by recombinant POPDC1 from myotubes was also confirmed by western blotting (Fig. 1c). (Uncropped blots shown in: Additional file 2).

New monoclonal antibodies against POPDC1 and POPDC2 Monoclonal antibodies were prepared against recombinant protein preparations and selected for their recognition of human POPDC1 or POPDC2 proteins using techniques described previously [28]. Immunofluorescence specificity of the new POPDC1 1C12 (Fig. 2a) and POPDC2 12G12 (Fig. 2b) mAbs was shown by the staining of COS7 cells transfected with recombinant POPDC1 or POPDC2 respectively. POPDC1 $1 \mathrm{C} 12$ did not recognise transfected POPDC2 and mAb POPDC2 12G12 did not recognise transfected POPDC1. Specificity of the mAbs was further demonstrated by competition experiments. Pre-incubation of mAb POPDC1 $1 \mathrm{C} 12$ with recombinant POPDC1, but not with recombinant POPDC2, inhibited localisation of the $\mathrm{mAb}$ to the sarcolemma of human skeletal muscle (Fig. 2c). Similarly, pre-incubation of mAb POPDC2 12G12 with recombinant POPDC2, but not with recombinant POPDC1, inhibited sarcolemmal localisation (Fig. 2d).

XIRP1 and POPDC1/2 are found, together with annexin A5, at intercalated discs and transverse tubules in rat or human heart sections

In rat heart, XIRP1 and POPDC 2 were both present at intercalated discs and near the Z-line of myofibrils
(Fig. 3a,b). Unlike XIRP1, however, POPDC2 was also present at the cardiac sarcolemma (Fig. 3b). A similar staining pattern in heart was reported by Alcalay et al. [8] for POPDC1 and Soni et al. [29] for POPDC2. Intercalated discs were identified using a connexin 43 antibody, which stains neither sarcolemma nor the Z-line (Fig. 3a). Our human-specific POPDC1 $\mathrm{mAb}$ does not recognize the rat protein, but a commercial POPDC1 antibody (BVES pAb STJ22848) gave similar results on rat heart to our POPDC2 mAb (Fig. 3c). Figure 3c also shows the location of POPDC1 near the Z-line which lies at the centre of the actin I-band stained by phalloidin (ALEXA 488 - phalloidin). This staining appears to correspond to transverse tubules, since an antibody against amphiphysin II, a marker for T-tubules, gave a similar staining pattern as POPDC2 (Fig. 3e) and POPDC proteins have transmembrane domains. Annexin A5 (ANXA5) was also present in both POPDC1 and POPDC2 pull downs (Table 1) and antibodies against this protein also located it close to the Z-line (Fig. 3d). Annexin A5 is a known component of the sarcolemma, transverse tubules and intercalated discs in the heart [30].

In human heart, mAbs POPDC1 1C12 (Fig. 4a) and POPDC2 12G12 (Fig. 4b) stained the intercalated discs and the cardiac sarcolemma whereas XIRP1 was present at the intercalated discs only. There were similar staining patterns in rat and human hearts, but cross striations were difficult to resolve in the human heart sections. In 


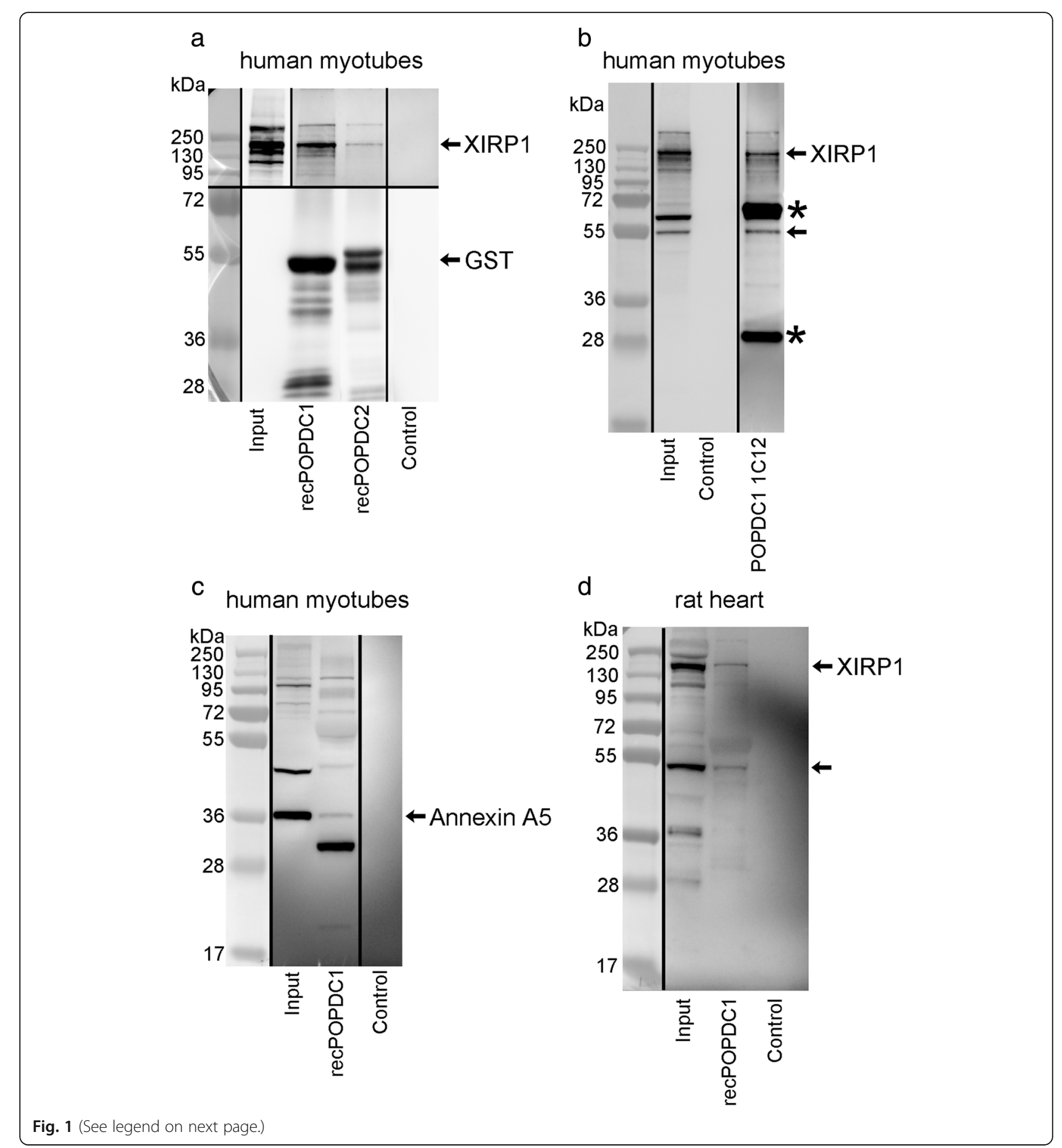


(See figure on previous page.)

Fig. 1 POPDC proteins pull down and co-immunoprecipitate XIRP1 and pull down annexin A5 from human myotube extract and pull-down XIRP1 from rat heart extract. Vertical black lines separate parts of blots that were originally non-adjacent or with different exposure times, as described. In all cases, the molecular weight markers in lane 1 were detected by colourimetric imaging and the antibody blots by chemiluminescence. a Glutathione beads were loaded with recombinant preparations of POPDC1, POPDC2 or not loaded (control for nonspecific binding) and incubated with human myotube extracts. "Input" shows human myotube extracts diluted to 20\%. Upper part of blot (A) (above $72 \mathrm{kDa}$ ) developed with mAb against XIRP1 (sc-166,658) shows XIRP1 in the input and pulled down by POPDC1 and POPDC2, but not in the control. The "Input" lane had a shorter exposure time than the others and the "Control" lane was originally non-adjacent. The Lower part of blot (A) (72 kDa and below) was from a separate but identical gel to the upper blot and was developed with mAb against GST tag (17A10) as a loading control. The "Control" lane was originally non-adjacent to the others. The predicted molecular weights of the Popdc1 and Popdc2 fusion proteins are 58 and $57 \mathrm{kDa}$ respectively, which are indicated approximately with a single arrow. b Pan mouse lgG Dynabeads were loaded with monoclonal antibody against POPDC1 (1C12) or not loaded (control) and incubated with human myotube extracts. The blot was developed with XIRP1 mAb and shows XIRP1 in the input and immunoprecipitated by the POPDC1 mAb but not by unloaded control beads. ${ }^{*}=$ mouse Ig $\mathrm{H}$ and $\mathrm{L}$ chains. Predicted molecular weights of human XIRP1, isoforms 1,2 and 3 are 199, 122 and $56 \mathrm{kDa}$ respectively. Arrows indicate bands corresponding to XIRP1 isoforms 1 and 3. The "POPDC1 1C12" lane had a longer exposure compared to the other lanes and the "molecular weight markers" were not originally in lane 1. c Glutathione beads loaded with recombinant POPDC1 or beads alone (control) were incubated with human myotube extract. The blot was developed with pAb against annexin A5. The arrow indicates a band corresponding to annexin A5 monomer $(36 \mathrm{kDa}$ ) in the input and POPDC1 pull-down, but absent from the non-specific binding control. The "Control" lane was originally nonadjacent to the others. $\mathbf{d}$ Glutathione beads loaded with recombinant POPDC1 or beads alone (control) were incubated with rat heart ventricle extract. The blot was developed with mAb against XIRP1. Arrows indicate bands corresponding to XIRP1 in the input and POPDC1 pull-down, but absent from the non-specific binding control. The original colourimetric and chemiluminescence images are shown in Additional File 2

low power microscopy (40x) of human heart, T-tubules were only faintly-stained (Fig. 4) compared with the higher power microscopy (63x) of rat heart (Fig. 3), suggesting that intercalated discs may be the major site of POPDC-XIRP1 interaction in the heart. Figures 3 and 4 show representative images of results that were obtained with sections from two different rat hearts and confirmed with the sections from a human heart.

POPDC1, POPDC2, XIRP1 and annexin A5 are also found in T-tubules in longitudinal sections of human skeletal muscle Figure 5a-d shows the co-localization of POPDC1 and 2, XIRP1 and annexin A5 at Z-lines/T-tubules in longitudinal sections of human skeletal muscle, using a monoclonal antibody against alpha-actinin to locate Z-lines (Fig. 5c).

\section{POPDC1, POPDC2 and XIRP1 are present at the sarcolemma in transverse sections of human skeletal muscle} POPDC1 (Fig. 6a) and POPDC2 (Fig. 6b) are present at the sarcolemma in transverse sections of skeletal muscle. XIRP1 is also present at the sarcolemma, but does not co-localise exactly with POPDC proteins. Figures 5 and 6 show representative images of sections from five different human muscle biopsies which gave similar results in these experiments.

\section{Discussion}

Our initial proteomic study of pull-downs from human myotube extracts using recombinant POPDC1 or POPDC2 as the bait proteins showed that XIRP1 and cardiac actin were the highest-scoring hits (Table 1). XIRP1 has been located to myotendinous junctions in skeletal muscle [22] and to intercalated discs in heart $[31,32]$. We examined the interacting POPDC and XIRP1 proteins further by co-localization studies, bearing in mind that POPDC proteins (via zonula occludens1, ZO-1 [33]) and XIRP1 [34] are known actin-binding proteins. Proteomic studies only give clues to potential interaction partners in a single tissue; Table 1 should not be expected to include all interaction partners of POPDC proteins and all potential partners revealed by proteomics require biochemical confirmation.

XIRP1 is a scaffolding protein which stabilizes and maintains the integrity of the intercalated disc. Overexpression of XIRP1 leads to increased phosphorylation of gap junction protein alpha 1 (GJA1), also known as connexin-43 (Cx43), and down-regulation of the activity of gap junctions in cardiomyocytes [35]). Knockout Xirp1 (or Xin $\alpha$ ) mice develop a late-onset cardiomyopathy with conduction defects [22]. Missense mutations in XIRP1 cause cardiac arrhythmias [24] and a number of mutations in XIRP1 that are classed as deleterious have been found in patients with conditions including myalgia, proximal weakness, arrhythmia, contractures and cardiac conduction defects [11]. XIRP proteins have binding domains for both $\beta$-catenin and p120-catenin, through which they attach to $\mathrm{N}$-cadherin in the intercalated disc [36]. Intercalated discs provide electromechanical coupling between adjacent cardiomyocytes. Depolarising current is transmitted via gap junctions in the intercalated discs from cell to cell across the heart. Defects in intercalated disc structure and function are common features of cardiomyopathy, arrhythmia and heart failure (Reviewed: [32]).

Annexin A5 was also a significant interaction partner for POPDC1/2 (Table 1). Annexin A5 is upregulated in failing human hearts [37] and has been linked to plasma 


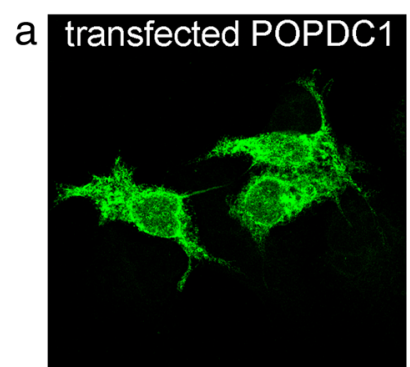

b
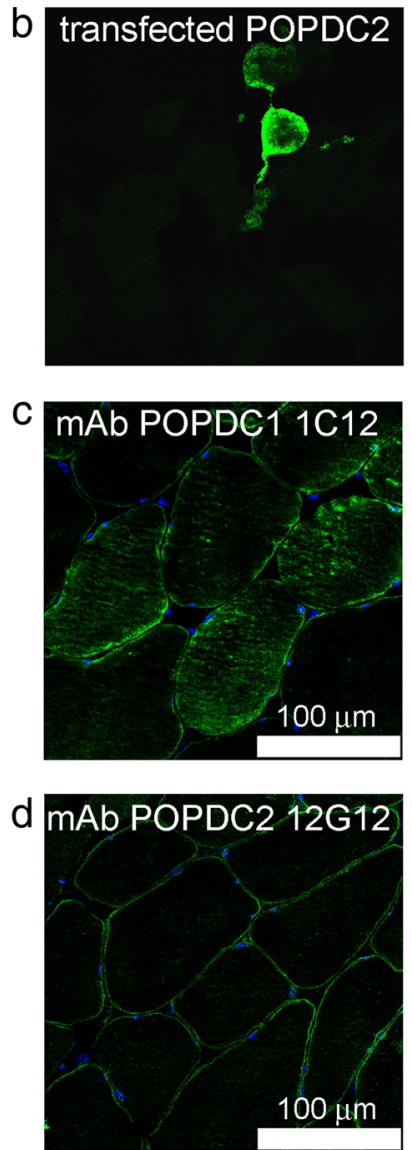
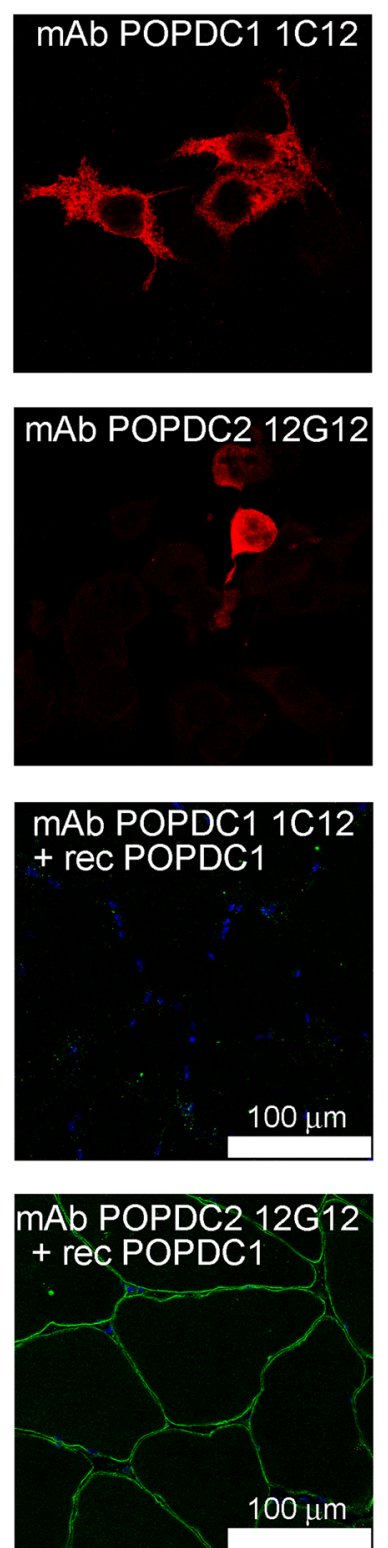
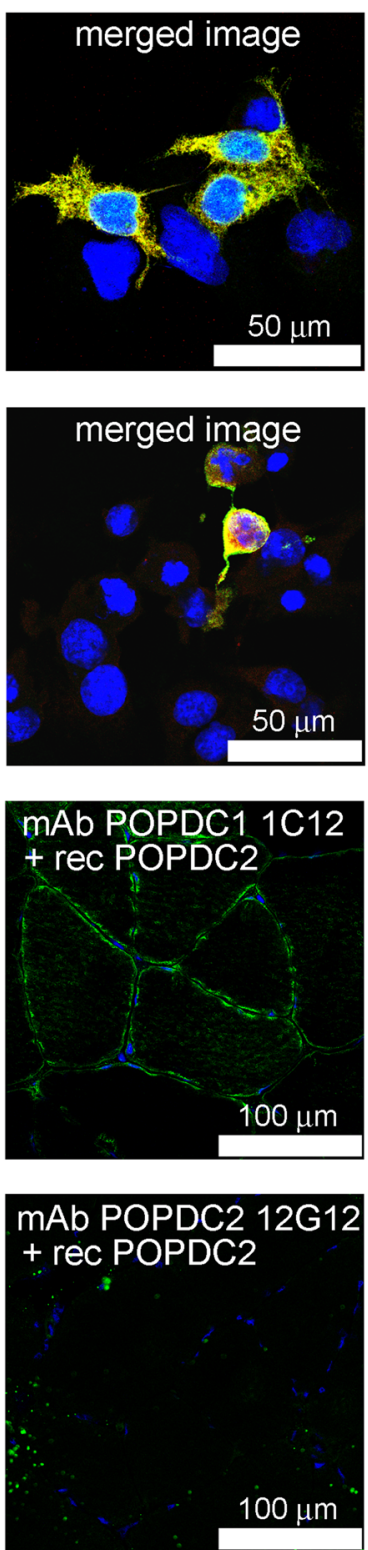

Fig. 2 Immunofluorescent specificity of POPDC mAbs. COS-7 cells were transfected with either (a) human POPDC1 (GFP tag) or (b) human POPDC2 (FLAG tag) and processed as described in "methods". a mAb POPDC1 1 C12 colocalised with transfected POPDC1 and (b) mAb POPDC2 12G12 colocalised with transfected POPDC2. The POPDC1 mAb did not recognise transfected POPDC2 and the POPDC2 mAb did not recognise transfected POPDC1 (not shown). c mAb POPDC1 1C12 localised to the sarcolemma of human skeletal muscle sections. This localisation was prevented by pre-incubation of the mAb with recombinant POPDC1 but not when the mAb was pre-incubated with recombinant POPDC2. Also, d localisation of mAb POPDC2 12G12 to the sarcolemma was not inhibited by pre-incubation with recombinant POPDC1 but this localisation was inhibited when the $\mathrm{mAb}$ was pre-incubated with recombinant POPDC2

membrane repair in skeletal muscle [38]), a property shared with caveolin-3 [39] and XIRP1 [40, 41]. There is some evidence of a role for POPDC1 in the maintenance of plasma membrane integrity, as discontinuities in the plasma membrane have been observed in the grandfather of a family carrying a pathogenic homozygous missense mutation (c.602C $>\mathrm{T}$, p.S201F) in POPDC1
[9]. This mutation gives rise to cardiac arrhythmia and LGMD [9].

Using new monoclonal antibodies (mAbs) specific for either POPDC1 (1C12) or POPDC2 (12G12), together with a commercial polyclonal antibody against POPDC1 (BVES), we have shown that both proteins are localized at the sarcolemma and transverse tubules in both human 


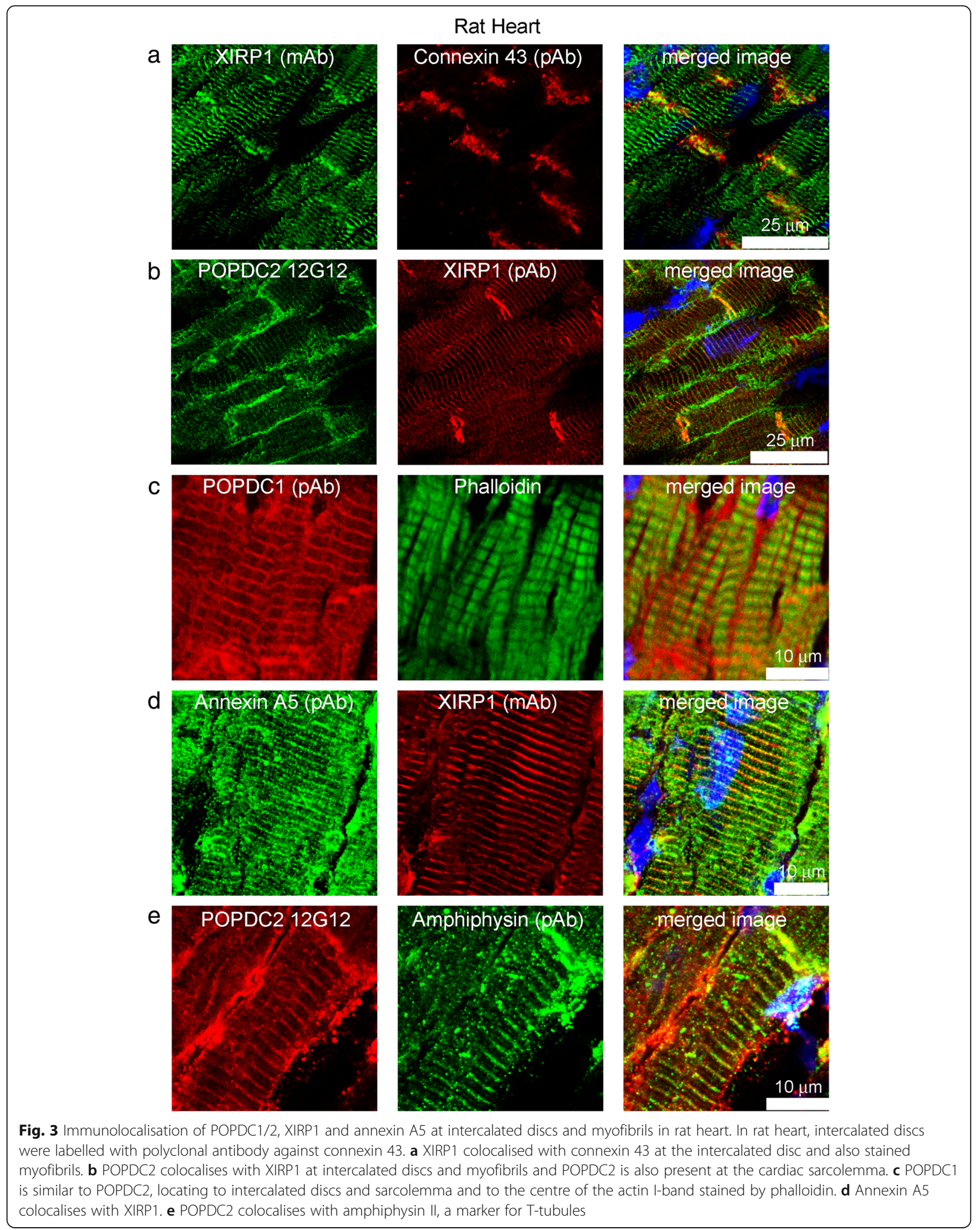




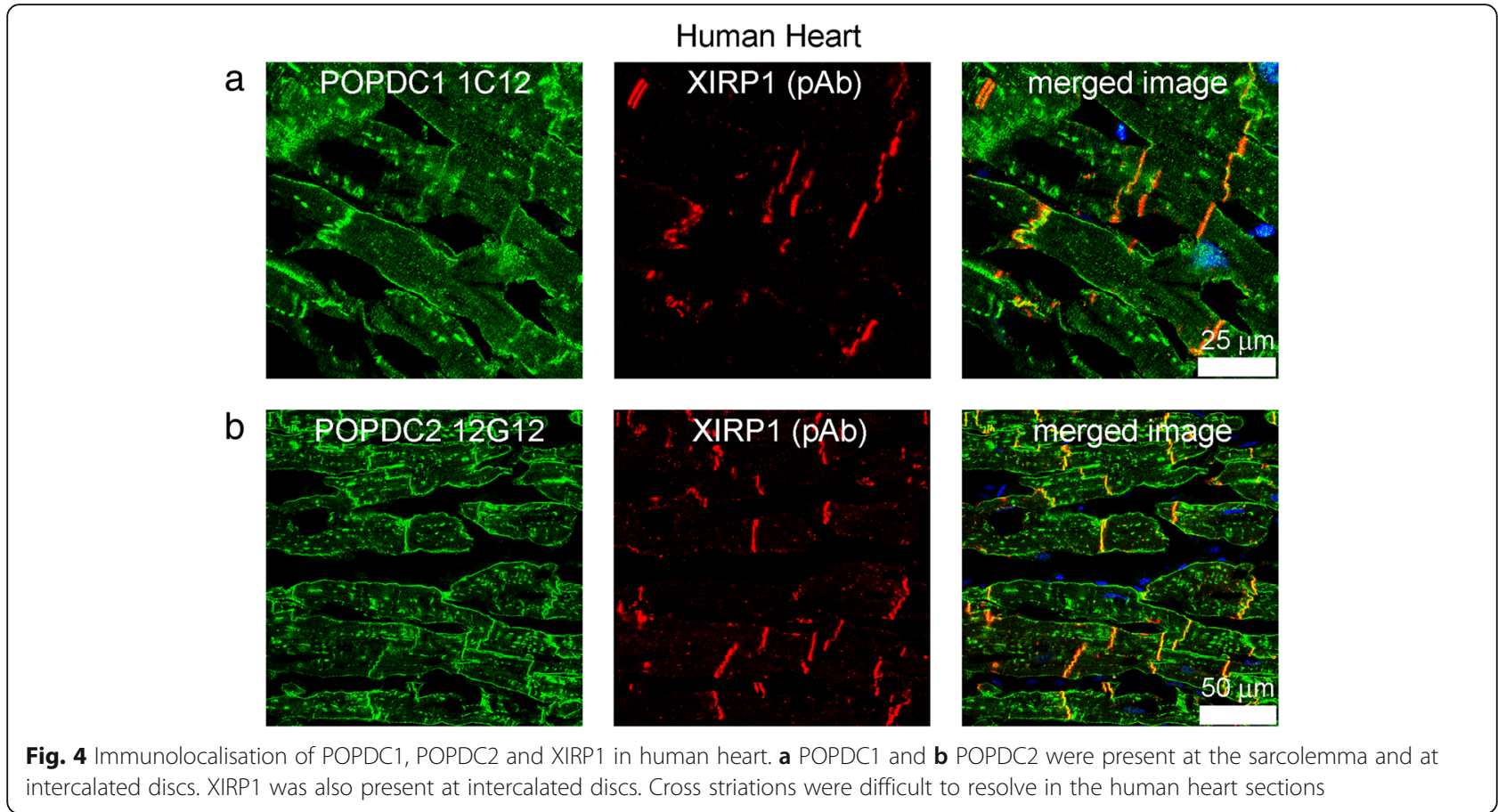

skeletal muscle and rat heart muscle. Partial co-localization of both XIRP1 and annexin A5 with POPDC1/2 was found in T-tubules in both cardiac and skeletal muscles. Ttubules are invaginations in the plasma membrane of muscle cells, they are rich in ion channels and relay excitation signals from the external membrane to the interior of cells and are very sensitive to eccentric stretch (reviewed in [42]). In heart, POPDC1 and 2 are mainly concentrated at intercalated discs. The presence of POPDC2 at intercalated discs was also shown by Soni et al. 2016 [29], while colocalization of XIRP1 with Connexin-43 at the intercalated discs of human hearts was shown by Xie et al. 2017 [35]. There is considerable functional overlap between POPDC proteins and XIRP proteins. In null mutants in the mouse for both gene families, a cardiac arrhythmia phenotype was reported. In the case of Popdc1 and Popdc2 null mutants, a stress-induced bradycardia phenotype was observed, while at baseline the mutants showed a normal rhythmic beating heart [7]. In contrast, the Xirp1 null mutant phenotype was characterized by a late-onset cardiomyopathy with conduction defects [22]. In patients, POPDC1 has also been linked to prolonged QT interval, increased QRS duration and AVblock [43]. Interestingly, in contrast to the mouse, in zebrafish, an AV-block was seen for both popdc1 and popdc2 null mutants $[9,17,43]$. Functional overlap is also seen in Popdc1 null mutants and Xirp1 null mutants with regard to skeletal muscle regeneration $[25,26]$.

Although the pull-downs clearly show association between POPDC1/2, XIRP1 and actin, it is not clear whether the interactions between them are direct or indirect. It is possible that they are members of a larger complex, some components of which were not detected by proteomics for technical reasons. Earlier studies identified different binding partners for POPDC proteins, including dystrophin, dysferlin, VAMP2, VAMP3, GEFT, NDRG4, ZO-1, TREK-1; c-MYC and PR61-alpha, as well as caveolin-3 [7-9, 33, 44-47]. None of these are among the 340 proteins that appeared in our pull-downs, although this may be either because their interaction with POPDC is tissue-specific and not occurring in myotube cultures, or because they are present at very low levels in RIPA buffer extracts, or because they showed some nonspecific binding to our control beads or because the interaction does not withstand the extraction conditions used. With numerous other interaction partners having been linked to POPDC1 and POPDC2 [4], it is not surprising that the loss-of-function phenotype of POPDC1 and XIRP1 would show only partial overlap. In this context it will also be interesting to define all components of the protein complex of which XIRP1 and POPDC1 are part in cardiac and skeletal muscle.

\section{Conclusions}

POPDC1/2 proteins interact with XIRP1 in skeletal myotubes and adult heart. Determining the functions of POPDC and XIRP1 proteins at the t-tubules and intercalated discs will help to understand their roles in normal cardiac and skeletal muscle function and what can go wrong when pathogenic mutations occur. 


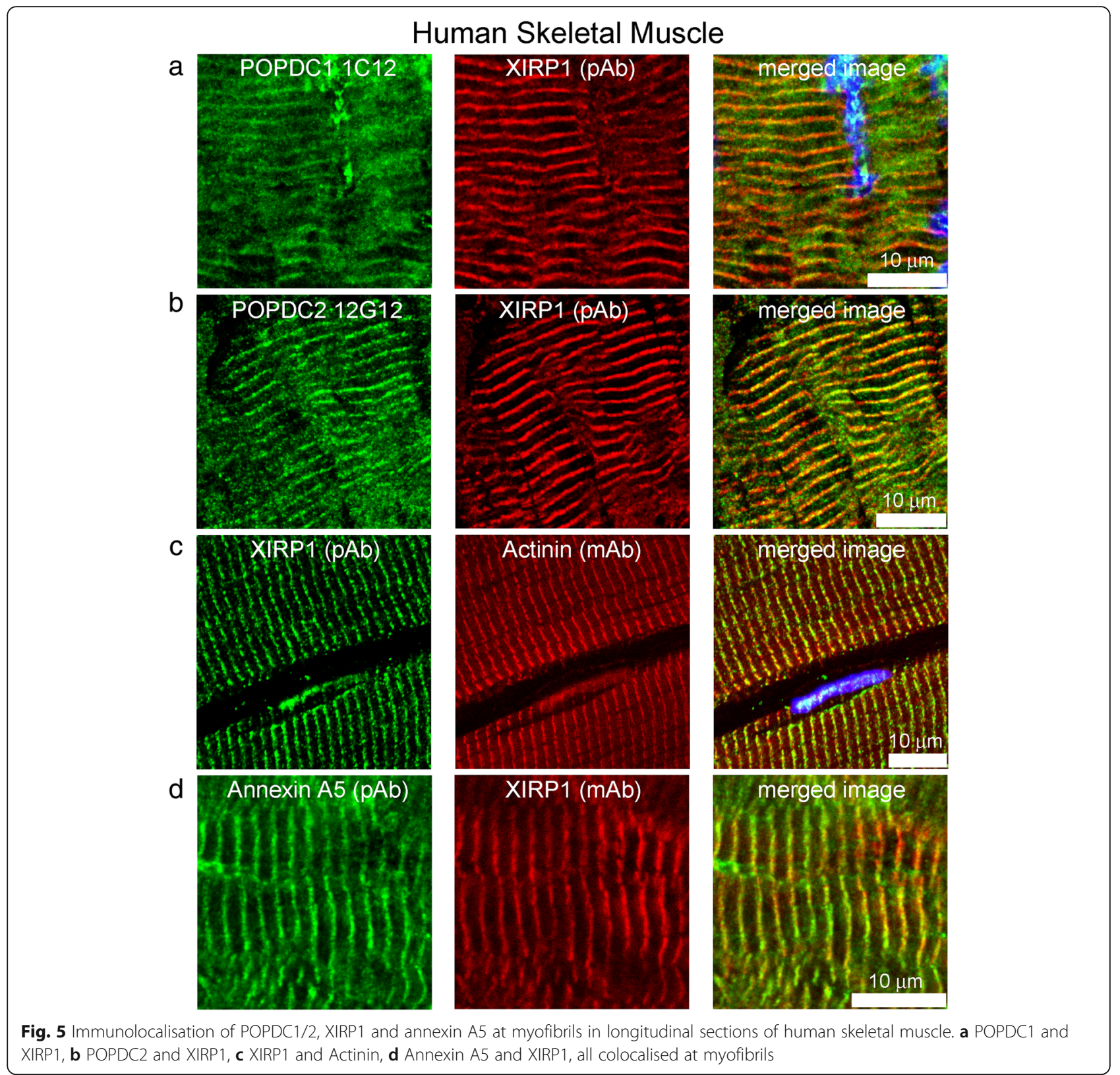

\section{Methods}

\section{Popdc "bait" proteins}

GST fusion proteins were generated by PCR from cDNA for mouse Popdc1 (Bves, GenBank Accession No: NM 024285.3) (aa 114 to 357) and for mouse Popdc2 (GenBank Accession No: NM_001081984.2) (aa 98 to 339) and cloned into pGEX bacterial expression vectors. pGEX constructs were transformed into $E$. coli BL21(DE3) and induced by IPTG to give GST-fusion proteins. After incubation the cells were washed with TNE buffer and sonicated sequentially with TNE, $2 \mathrm{M}$ urea, $4 \mathrm{M}$ urea, $6 \mathrm{M}$ urea and $8 \mathrm{M}$ urea. A Coomassie Blue-stained gel showed that most of the recombinant proteins were extracted in the $6 \mathrm{M}$ urea fraction and these were used in subsequent work. Resultant proteins contained GST-tags followed by the intracellular C-terminal tails of mPopdc1 and mPopdc2, with molecular weights of 58.3 and $57.1 \mathrm{kDa}$ respectively.

\section{Muscle cell culture and extraction}

A clonal immortalized human myoblast cell line from a 25-year-old control donor without neuromuscular disease was immortalized by transduction with human telomerase reverse transcriptase (hTERT) and cyclindependent kinase-4 (Cdk4) containing retroviral vectors, at the Institut de Myologie, Paris, as described previously [48]. Myoblasts were cultured in skeletal muscle cell growth medium (Cat No: C-23060; PromoCell GmbH, Heidelberg, Germany) containing supplement mix (Cat 


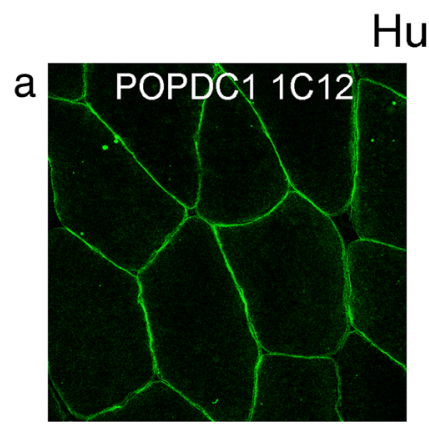

\section{Human Skeletal Muscle}
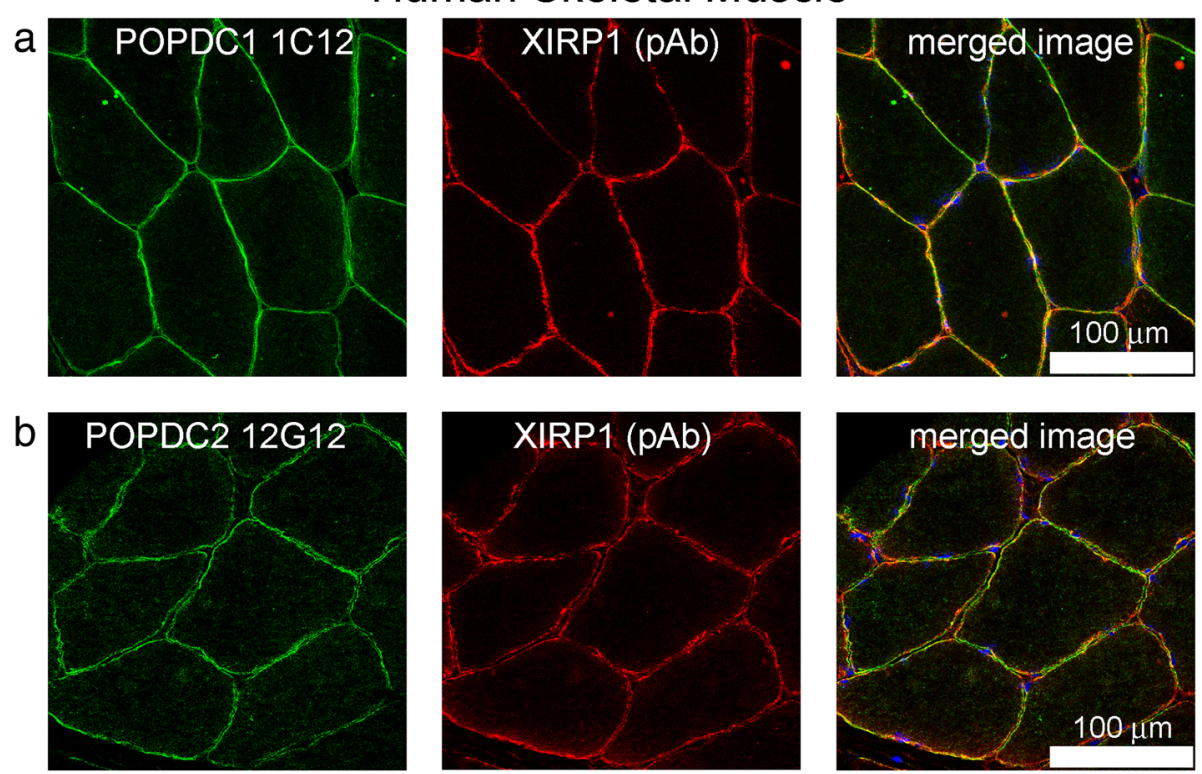

Fig. 6 Immunolocalisation of POPDC1/2 and XIRP1 in transverse section of human skeletal muscle. a POPDC1 mAb 1C12 and b POPDC2 mAb 12G12 showed largely even and regular sarcolemmal staining whereas XIRP1 was often discontinuous at the sarcolemma

No: C-39365; PromoCell) with 20\% Fetal Bovine Serum (Cat No: 10270; Gibco; ThermoFisher Scientific, Paisley, UK). At around $80 \%$ confluency, adherent myoblasts were washed in medium lacking serum and differentiation was induced by culturing in DMEM (Cat No: 31966-021; Gibco; ThermoFisher Scientific) supplemented with Insulin $(1721 \mathrm{nM})$, Transferrin $(68.7 \mathrm{nM})$, Selenium (38.7 nM) (ITS-X; Cat No: 51500-056; Gibco; ThermoFisher Scientific) and Penicillin-Streptomycin (Cat No: DE17-603E; Lonza; Verviers, Belgium). Cell culture was continued for a further 4 days, after which, over $80 \%$ of the cells had fused into myotubes. Cells cultured for pull-down experiments were extracted in RIPA buffer (1\% NP-40; $0.25 \%$ sodium deoxycholate; $1 \mathrm{mM}$ EDTA; $150 \mathrm{mM} \mathrm{NaCl} ; 50 \mathrm{mM}$ Tris- $\mathrm{HCl}, \mathrm{pH} 7.4$ ), in the presence of protease inhibitor cocktail (Sigma P8340; plus $1 \mathrm{mM}$ PMSF), by sonication followed by centrifugation.

\section{Pull-down and proteomic analysis}

Glutathione high capacity magnetic agarose beads (Cat No: G0924; Sigma) were loaded with GST-tagged "Bait" proteins (mouse Popdc1 or mouse Popdc2 or No Bait control) and then incubated with "Prey" (cultured human myotube extract in RIPA buffer). Captured proteins were digested by overnight incubation with $1 \% \mathrm{v} / \mathrm{v}$ tryp$\sin$ at $37^{\circ} \mathrm{C}$ and analysed by nanoLC MSMS as described previously [49]. The MS/MS data file was analysed using the Mascot search algorithm (Matrix Science), against the SwissProt database (May 2018) restricting the search to Homo sapiens $(557,491$ sequences), with trypsin as the cleavage enzyme and methionine oxidation as a variable modification. The peptide mass tolerance was set to $20 \mathrm{ppm}$ and the MSMS mass tolerance to $\pm 0.1 \mathrm{Da}$. This analysis was used to identify medium-to-high abundance proteins that interact with Popdc proteins.

\section{Extraction and pull-down with rat heart}

The heart was removed from a 5 month old Sprague Dawley rat that had been euthanized humanely with an overdose of pentobarbitone anesthetic $(0.5 \mathrm{~mL} / 100 \mathrm{~g})$ via intraperitoneal injection. The heart was cut in half, washed with PBS, weighed, snap frozen in liquid nitrogen, transferred to a mortar and ground to a fine powder with a pestle. The liquid nitrogen was then allowed to evaporate and the powdered heart extracted with RIPA buffer with inhibitors (around $1 \mathrm{~mL}$ buffer per $100 \mu \mathrm{g}$ heart), sonicated on ice and centrifuged. The heart extract was used instead of myotube extract as "prey", with mouse GST- mouse Popdc1 on glutathione beads as "bait", as described earlier.

\section{Hybridoma production}

Human BVES (POPDC1) cDNA (GenBank Accession No: NM_007073.4) was a gift from D. Bader [33]. A GST fusion protein was generated by PCR from the Cterminal tail of (aa 115-347) and cloning into the pGEX bacterial expression vector. This protein contains the GST tag followed by the intracellular C-terminal tail of POPDC1. The GST-mouse Popdc2 (amino acids 98 to 339) fusion protein preparation described earlier for 
pull-down experiments was also used for hybridoma production. Induction and extraction of the GST-fusion proteins was performed as described earlier. Fusion protein in the $6 \mathrm{M}$ urea fraction was used in the protocol for immunization of $\mathrm{BALB} / \mathrm{c}$ mice for production of mAbs [28]. ELISA plates were coated with either the GST-POPDC fusion proteins used for immunization or with an unrelated GST-fusion protein, in order to eliminate those mAbs reacting with the GST fusion tag. Hybridoma supernatants were additionally screened for colocalisation with transfected human POPDC proteins as follows: COS-7 cells were transfected (Lipofectamine) with either hPOPDC1-YFP or hPOPDC2-FLAG tag on glass coverslips and the cells fixed and permeabilized with $50 \%$ acetone: $50 \%$ methanol. Transfected cells were incubated with hybridoma supernatants. hPOPDC1-YFP transfectants were then incubated with $5 \mu \mathrm{g} / \mathrm{ml}$ goat anti-mouse ALEXA 546 (Cat No: A11030, Molecular Probes, Eugene, Oregon, USA). hPOPDC2-FLAG transfectants were incubated with rabbit oligoclonal antiFLAG (Cat No: 740001, Thermo Fisher Scientific), followed by $5 \mu \mathrm{g} / \mathrm{ml}$ goat anti-mouse ALEXA 546 (Cat No: A11030, Molecular Probes) and $5 \mu \mathrm{g} / \mathrm{ml}$ goat antirabbit ALEXA 488 (Cat No: A11034, Molecular Probes). DAPI was added for the final $10 \mathrm{~min}$ of incubation to counterstain nuclei and confocal microscopy performed as described below. Monoclonal antibodies POPDC1$1 \mathrm{C} 12$ and POPDC2-12G12 were selected because they recognized the corresponding transfected POPDC1 or POPDC2 by immunofluorescence microscopy (Fig. 2).

\section{SDS-polyacrylamide gel electrophoresis and Western blotting}

Samples were mixed with SDS buffer $(125 \mathrm{mM}$ Tris $\mathrm{pH}$ 6.8; $2 \%$ SDS; 5\% 2-beta mercaptoethanol; 5\% glycerol; with bromophenol blue), boiled and subjected to SDSPAGE using $10 \%$ polyacrylamide gels and transferred to nitrocellulose membranes (Protan BA85, Whatman). Non-specific sites were blocked with $5 \%$ skimmed milk protein, membranes washed with PBS and then incubated with primary monoclonal antibodies against: XIRP1, (Xin-alpha (D-8); sc-166,658; 1/100; Santa Cruz Biotechnology, Insight Biotechnology Ltd., Wembly, UK); or GST, mAb 17A10 (1/100). This was followed by washing in PBS and incubation with secondary antibody (peroxidase-labelled rabbit anti-mouse immunoglobulins, P0260; 1/1000; Dako, Denmark). Alternatively, for the detection of annexin A5, pAb ANXA5 (Abcam; ab14196; $1.4 \mu \mathrm{g} / \mathrm{mL}$ ) primary antibody followed by goat anti-rabbit Ig HRP (P0448; Dako; 1/1000) secondary antibody. All antibodies were diluted in PBS containing $0.05 \%$ Triton X, 0.1\% BSA, $1 \%$ horse serum and $1 \%$ fetal bovine serum. XIRP1 and annexin A5 antibody reacting bands were detected with SuperSignal West Femto chemiluminescent reagent (Cat No: 34094; ThermoFisher Scientific) and GST antibody reacting bands were detected with SuperSignal West Pico Plus chemiluminescent reagent (Cat No: 34580; ThermoFisher Scientific) and visualized with a ChemiDoc Touch imaging system (BioRad Ltd.).

\section{Immunoprecipitation}

Superparamagnetic polystyrene beads coated with monoclonal human anti-mouse IgG antibodies (Dynabeads Pan Mouse IgG; Cat No: 11041; Invitrogen) were washed with $4 \%$ bovine serum albumin in PBS and then incubated with $\mathrm{mAb}$ against POPDC1 (1C12) or PBS alone (control) with gentle rolling for $1 \mathrm{~h}$. Beads were then washed with PBS and incubated with cultured human myotube extract in RIPA buffer for $1 \mathrm{~h}$ with rolling. Beads were thoroughly washed with RIPA buffer and then with PBS and the bound material extracted by incubating the beads with SDS sample buffer, heating to $90^{\circ} \mathrm{C}$ for $3 \mathrm{~min}$ and then saving the eluate.

\section{Immunofluorescence microscopy}

Immunohistochemistry was performed on unfixed cryostat sections of human skeletal muscle or rat cardiac ventricle muscle. Monoclonal antibodies against: POPDC1 (1C12; 1: 2 dilution); POPDC2 (12G12 1:2 dilution); XIRP1 (Xinalpha (D-8); sc-166,658; 1/100); Actinin (MANDYS 141, 4A12, 1/50 [50]); were diluted and incubated on specimens for $1 \mathrm{~h}$. Specimens were then washed and incubated with rabbit polyclonal antibodies: Connexin 43 (GJA1, ab11370; Abcam; 1/1000); XIRP1 (Cat No: HPA016750; Sigma; 1/ 150); Annexin A5 (ANXA5, ab14196; Abcam; 1/50); POPDC1/BVES (Cat No: STJ22848; St. John's Laboratory Ltd., London, E16 2RD; 1/100); Amphiphysin (ab244375; Abcam; 1/50). Following antibody incubation, specimens were washed with PBS and then incubated with $5 \mu \mathrm{g} / \mathrm{ml}$ of each secondary antibody which was either: goat anti-mouse ALEXA 488 (Cat No: A11029, Molecular Probes, Eugene, Oregon, USA) and goat anti-rabbit ALEXA 546 (Cat No: A11010), or with goat anti-mouse ALEXA 546 (Cat No: A11030) and goat anti-rabbit ALEXA 488 (Cat No: A11034). Secondary antibodies were diluted in PBS containing $1 \%$ horse serum, $1 \%$ fetal bovine serum and $0.1 \%$ $\mathrm{BSA}$, and incubated on the sections for $1 \mathrm{~h}$. Alternatively, for some immunofluorescent labelling of rat cardiac sections, a single primary (polyclonal) antibody then single goat anti-rabbit ALEXA 546 antibody were used, followed by ALEXA-488 Phalloidin (Cat No: A12383; Molecular Probes) to label filamentous actin. Finally, DAPI (at $200 \mathrm{ng} /$ $\mathrm{ml}$ ) was added for the final $10 \mathrm{~min}$ of incubation to counterstain nuclei before washing and mounting the sections (Hydromount; Merck). For protein competition experiments, monoclonal antibodies were pre-incubated with 1 $\mathrm{mg} / \mathrm{mL}$ recombinant protein preparation for $1 \mathrm{~h}$ at room 
temperature. Images were acquired by sequential scanning with a Leica TCS SP5 spectral confocal microscope (63x/ 1.4 oil) (Leica Microsystems, Milton Keynes, UK), except the human heart images which were acquired with a Zeiss LSM780 confocal microscope (40x/1.3 oil) (Carl Zeiss Microscopy, Cambridge, UK.

\section{Supplementary Information}

The online version contains supplementary material available at https://doi. org/10.1186/s12860-020-00329-3.

Additional file 1. Table; Unfiltered mass spectrometry results.

Additional file 2. Original uncropped blots used for Fig. 1.

\section{Abbreviations}

ACTC1: Actin Alpha Cardiac Muscle 1; ANXA5: Annexin A5;

AV: Atrioventricular; BIN1: Bridging Integrator 1 (also known as Amphiphysin II); BSA: Bovine serum albumin; BVES: Blood Vessel Epicardial Substance (also known as POPDC 1); CAMP: Cyclic adenosine 3',5'-monophosphate; CAV3: Caveolin 3; Cdk4: Cyclin-dependent kinase-4; CMYA1: Cardiomyopathyassociated protein 1 (also known as XIRP1); C-Myc: MYC Proto-Oncogene; COS-7: African green monkey kidney fibroblast-like cell line; Cx43: Connexin43 (Gap Junction Protein Alpha 1, GJA1); DAPI: 4',6-diamidino-2-phenylindole; FLAG: DYKDDDDK-tag; FRET: Fluorescence resonance energy transfer; GEFT: ARHGEF25 or Rho Guanine Nucleotide Exchange Factor 25: GST: Glutathione S-transferase; HEK293: Human embryonic kidney 293 cells; HRP: Horseradish peroxidase; hTERT: Human telomerase reverse transcriptase; IPTG: Isopropyl beta-D-1-thiogalactopyranoside; KCNK2: Potassium Two Pore Domain Channel Subfamily K Member 2 (TREK-1); LGMD: Limb-girdle muscular dystrophy; mAb: Monoclonal antibody; Nano LC MSMS: Nanoscale liquid chromatography tandem mass spectrometry; NDRG4: NDRG Family Member 4 (or N-Myc Downstream-Regulated Gene 4 Protein); pAb: Polyclonal antibody; PBS: Phosphate-buffered saline; PCR: Polymerase chain reaction; PMSF: Phenylmethylsulfonyl fluoride (serine protease inhibitor); POPDC1: Popeye Domain Containing 1 (also known as BVES); POPDC2: Popeye Domain Containing 2; POPDC3: Popeye Domain Containing 3; PR61-alpha: Protein Phosphatase 2 Regulatory Subunit B'Alpha (PPP2R5A); RIPA buffer: Radioimmunoprecipitation assay buffer; SDS: Sodium dodecyl sulfate; TNE buffer: Tris-HCl, NaCl, EDTA buffer; TREK-1: Potassium Two Pore Domain Channel Subfamily K Member 2 (KCNK2); VAMP2: Vesicle Associated Membrane Protein 2; VAMP3: Vesicle Associated Membrane Protein 3; XIRP1: Xin Actin Binding Repeat Containing 1 (also known as XIN or CMYA1 - cardiomyopathy-associated protein 1); YFP: Yellow fluorescent protein; ZO-1: Zonula Occludens Protein 1 (Tight Junction Protein 1)

\section{Acknowledgements}

We thank The Platform for Immortalization of Human Cells, Institute of Myology, Paris, France, for the immortalized human myoblasts. We thank Ursula Herbort-Brand for expert technical assistance. The donation of the POPDC1-GST construct by David Bader is gratefully acknowledged.

\section{Authors' contributions}

Conceived and designed the experiments: IH, HRF, TB, GEM; Hybridoma production: IH, GEM; Mass spectrometry: HRF, SLS; Other experimental work: IH, HRF, RFRS, GEM; Analysed the data: IH, HRF, RFRS, SLS, TB, GEM; Wrote the manuscript: IH, HRF, TB, GEM. All authors read and approved the final manuscript.

\section{Funding}

This work was supported by the British Heart Foundation [PG/16/68/31991; to IH and GEM], [PG/19/13/34247; to TB] and the Orthopaedic Institute Ltd., RJAH Orthopaedic Hospital, Oswestry UK [RPG141 and RPG189; to IH and GEM]. Infrastructure support for this research was provided by the NIHR Imperial Biomedical Research Centre (BRC) and ICHTB. The funding bodies played no role in the design of the study and collection, analysis, and interpretation of data and in writing the manuscript.

\section{Availability of data and materials}

Mass spectrometry data generated and analysed during this study are available in the supplementary information file, "Additional file 1". Monoclonal antibodies are available from the MDA monoclonal antibody resource, Oswestry, UK: http://www.glennmorris.org.uk/mabs/WCIND.htm

POPDC nucleotide sequence data is available from GenBank with the following accession numbers: Mouse Bves/Popdc1 (NM_024285.3); Mouse Popdc2 (NM_001081984.2); Human BVES/POPDC1 (NM_007073.4); Human POPDC2 (NM_022135.4).

\section{Ethics approval and consent to participate}

This study has been approved by the RJAH Orthopaedic Hospital Research Committee. All human tissue studies complied with the UK Human Tissue Act (2006). Human biopsies were obtained following written informed consent using protocols approved by RJAH Orthopaedic Hospital, Oswestry and Imperial College, London. Muscle biopsy for immortalisation of myoblasts was obtained with written informed consent in accordance with European recommendations and French legislation. All animal procedures were performed with approval of the Keele University Animal Welfare and Ethical Review Body and conducted under the licensed authority of the UK Home Office.

\section{Consent for publication}

Not applicable.

\section{Competing interests}

The authors declare that they have no competing interests.

\section{Author details}

${ }^{1}$ Wolfson Centre for Inherited Neuromuscular Disease, RJAH Orthopaedic Hospital, Oswestry SY10 7AG, UK. ${ }^{2}$ School of Pharmacy and Bioengineering, Keele University, Keele ST5 5BG, UK. ${ }^{3}$ Imperial Centre of Translational and Experimental Medicine, National Heart and Lung Institute, Imperial College, 4th Floor, Du Cane Road, London W12 ONN, UK. ${ }^{4}$ BSRC Mass Spectrometry and Proteomics Facility, University of St Andrews, North Haugh, St Andrews, Fife KY16 9ST, UK.

Received: 8 July 2020 Accepted: 18 November 2020

Published online: 01 December 2020

\section{References}

1. Reese DE, Zavaljevski M, Streiff NL, Bader D. Bves: A novel gene expressed during coronary blood vessel development. Dev Biol. 1999;209:159-71.

2. Andrée B, Hillemann T, Kessler-Icekson G, Schmitt-John T, Jockusch H, Arnold HH, Brand T. Isolation and characterization of the novel popeye gene family expressed in skeletal muscle and heart. Dev Biol. 2000;223: 371-82.

3. Brand T, Simrick SL, Poon KL, Schindler RF. The CAMP-binding Popdc proteins have a redundant function in the heart. Biochem Soc Trans. 2014; 42:295-301.

4. Brand T. POPDC proteins and cardiac function. Biochem Soc Trans. 2019;47: 1393-404.

5. Knight RF, Bader DM, Backstrom JR. Membrane topology of Bves/Pop1A, a cell adhesion molecule that displays dynamic changes in cellular distribution during development. J Biol Chem. 2003;278:32872-9.

6. Vasavada TK, DiAngelo JR, Duncan MK. Developmental expression of Pop1/ Bves. J Histochem Cytochem. 2004:52:371-7.

7. Froese A, Breher SS, Waldeyer C, Schindler RF, Nikolaev VO, Rinné S, Wischmeyer E, Schlueter J, Becher J, Simrick S, et al. Popeye domain containing proteins are essential for stress-mediated modulation of cardiac pacemaking in mice. J Clin Invest. 2012;122:1119-30.

8. Alcalay Y, Hochhauser E, Kliminski V, Dick J, Zahalka MA, Parnes D, Schlesinger $H$, Abassi Z, Shainberg A, Schindler RF, et al. Popeye domain containing 1 (Popdc1/Bves) is a caveolae-associated protein involved in ischemia tolerance. PLoS One. 2013;8:e71100.

9. Schindler RF, Scotton C, Zhang J, Passarelli C, Ortiz-Bonnin B, Simrick S, Schwerte T, Poon KL, Fang M, Rinné S, et al. POPDC1 (S201F) causes muscular dystrophy and arrhythmia by affecting protein trafficking. J Clin Invest. 2016;126:239-53. 
10. De Ridder W, Nelson I, Asselbergh B, De Paepe B, Beuvin M, Ben Yaou R, Masson C, Boland A, Deleuze JF, Maisonobe T, et al. Muscular dystrophy with arrhythmia caused by loss-of-function mutations in BVES. Neurol. Genet. 2019:e321:5.

11. Meinke P, Kerr ARW, Czapiewski R, de Las Heras Jl, Dixon CR, Harris E, Kölbel $H$, Muntoni F, Schara U, Straub V, et al. A multistage sequencing strategy pinpoints novel candidate alleles for Emery-Dreifuss muscular dystrophy and supports gene misregulation as its pathomechanism. EBioMedicine. 2020:102587:51.

12. Rinné S, Ortiz-Bonnin B, Stallmeyer B, Schindler RFR, Kiper AK, Dittmann S, Friedrich C, Zumhagen S, Simrick SL, González W, et al. Conduction disorder caused by a mutation in POPDC2, a novel modulator of the cardiac sodium channel SCN5A. Acta Physiol. 2016;216(S707):0S03-1 (Abstract).

13. Vissing J, Johnson K, Töpf A, Nafissi S, Díaz-Manera J, French VM, Schindler RF, Sarathchandra P, Løkken N, Rinné S, et al. POPDC3 Gene Variants Associate with a New Form of Limb Girdle Muscular Dystrophy. Ann Neurol. 2019;86:832-43.

14. Gingold-Belfer $R$, Bergman M, Alcalay $Y$, Schlesinger $H$, Aravot $D$, Berman $M$, Salman H, Brand T, Kessler-lcekson G. Popeye domain-containing 1 is downregulated in failing human hearts. Int J Mol Med. 2011;27:25-31.

15. Andrée B, Fleige A, Arnold HH, Brand T. Mouse Pop1 is required for muscle regeneration in adult skeletal muscle. Mol Cell Biol. 2002;22:1504-12.

16. Boukens BJ, Christoffels VM. Popeye proteins: muscle for the aging sinus node. J Clin Invest. 2012;122:810-3.

17. Kirchmaier BC, Poon KL, Schwerte T, Huisken J, Winkler C, Jungblut B, Stainier DY, Brand T. The Popeye domain containing 2 (popdc2) gene in zebrafish is required for heart and skeletal muscle development. Dev Biol. 2012;363:438-50 Erratum in: Dev Biol. 366,433.

18. Schindler RF, Scotton C, French V, Ferlini A, Brand T. The Popeye Domain Containing Genes and their Function in Striated Muscle. J Cardiovasc Dev Dis. 2016:3:22.

19. Parang B, Thompson JJ, Williams CS. Blood Vessel Epicardial Substance (BVES) in junctional signaling and cancer. Tissue Barriers. 2018;6:1-12.

20. Han P, Lei Y, Li D, Liu J, Yan W, Tian D. Ten years of research on the role of BVES/ POPDC1 in human disease: a review. Onco Targets Ther. 2019;12: 1279-91.

21. Amunjela JN, Swan AH, Brand T. The role of the Popeye domain containing gene family in organ homeostasis. Cells. 2019;8:1594.

22. Feng HZ, Wang Q, Reiter RS, Lin JL, Lin JJ, Jin JP. Localization and function of Xina in mouse skeletal muscle. Am J Phys Cell Phys. 2013;304:C1002-12.

23. Wang Q, Lin JL, Chan SY, Lin JJ. The Xin repeat-containing protein, mXin $\beta$, initiates the maturation of the intercalated discs during postnatal heart development. Dev Biol. 2013;374:264-80.

24. Huang L, Wu KH, Zhang L, Wang Q, Tang S, Wu Q, Jiang PH, Lin JJ, Guo J, Wang $L$, et al. Critical Roles of Xirp Proteins in Cardiac Conduction and Their Rare Variants Identified in Sudden Unexplained Nocturnal Death Syndrome and Brugada Syndrome in Chinese Han Population. J Am Heart Assoc. 2018; 7:e006320

25. Al-Sajee D, Nissar AA, Coleman SK, Rebalka IA, Chiang A, Wathra R, van der Ven PF, Orfanos Z, Hawke TJ. Xin-deficient mice display myopathy, impaired contractility, attenuated muscle repair and altered satellite cell functionality. Acta Physiol (Oxford). 2015;214:248-60.

26. Nissar AA, Zemanek B, Labatia R, Atkinson DJ, van der Ven PF, Fürst DO, Hawke TJ. Skeletal muscle regeneration is delayed by reduction in Xin expression: consequence of impaired satellite cell activation? Am J Phys Cell Phys. 2012;302:C220-7

27. Alonso S, Garner I, Vandekerckhove J, Buckingham M. Genetic analysis of the interaction between cardiac and skeletal actin gene expression in striated muscle of the mouse. J Mol Biol. 1990;211:727-38.

28. Nguyen TM, Morris GE. A rapid method for generating large numbers of high-affinity monoclonal antibodies from a single mouse. In: Walker JM, editor. The Protein Protocols Handbook. 3rd ed. Totowa NJ: Humana Press; 2010. p. 1961-74.

29. Soni S, Raaijmakers AJ, Raaijmakers LM, Damen JM, van Stuijvenberg L, Vos MA, Heck AJ, van Veen TA, Scholten A. A Proteomics Approach to Identify New Putative Cardiac Intercalated Disk Proteins. PLoS One. 2016;11: e0152231.

30. Benevolensky D, Belikova Y, Mohammadzadeh R, Trouvé P, Marotte F, Russo-Marie F, Samuel $J$, Charlemagne D. Expression and Localization of the Annexins II, V, and VI in Myocardium From Patients With End-Stage Heart Failure. Lab Investig. 2000;80:123-33.
31. Palatinus JA, Gourdie RG. Xin and the art of intercalated disk maintenance. Am J Physiol Heart Circ Physiol. 2007;293:H2626-8.

32. Wang Q, Lin JL, Erives AJ, Lin Cl, Lin JJ. New insights into the roles of Xin repeat-containing proteins in cardiac development, function, and disease. Int Rev Cell Mol Biol. 2014;310:89-128.

33. Osler ME, Chang MS, Bader DM. Bves modulates epithelial integrity through an interaction at the tight junction. J Cell Sci. 2005;118:4667-78.

34. Jung-Ching Lin J, Gustafson-Wagner EA, Sinn HW, Choi S, Jaacks SM, Wang DZ, Evans S, Li-Chun Lin J. Structure, Expression, and Function of a Novel Intercalated Disc Protein. Xin J Med Sci. 2005;25:215-22.

35. Xie Y, Liu S, Hu S, Wei Y. Cardiomyopathy-Associated Gene 1-Sensitive PKCDependent Connexin 43 Expression and Phosphorylation in Left Ventricular Noncompaction Cardiomyopathy. Cell Physiol Biochem. 2017:44:828-42.

36. Wang Q, Lu TL, Adams E, Lin JL, Lin JJ. Intercalated disc protein, mXina, suppresses p120-catenin-induced branching phenotype via its interactions with p120-catenin and cortactin. Arch Biochem Biophys. 2013;535:91-100.

37. Matteo RG, Moravec CS. Immunolocalization of annexins IV, $V$ and $V I$ in the failing and non-failing human heart. Cardiovasc Res. 2000;45:961-70.

38. Carmeille R, Bouvet F, Tan S, Croissant C, Gounou C, Mamchaoui K, Mouly V, Brisson AR, Bouter A. Membrane repair of human skeletal muscle cells requires Annexin-A5. Biochim Biophys Acta. 2016;1863:2267-79.

39. Cai C, Weisleder N, Ko JK, Komazaki S, Sunada Y, Nishi M, Takeshima H, Ma J. Membrane repair defects in muscular dystrophy are linked to altered interaction between MG53, caveolin-3, and dysferlin. J Biol Chem. 2009;284: 15894-902.

40. Hawke TJ, Atkinson DJ, Kanatous SB, Van der Ven PFM, Goetsch SC, Garry DJ. Xin, an Actin Binding Protein, Is Expressed Within Muscle Satellite Cells and Newly Regenerated Skeletal Muscle Fibers. Am J Phys Cell Phys. 2007; 293:C1636-44

41. Otten C, van der Ven PF, Lewrenz I, Paul S, Steinhagen A, Busch-Nentwich E, Eichhorst J, Wiesner B, Stemple D, Strähle U, et al. Xirp proteins mark injured skeletal muscle in zebrafish. PLoS One. 2012;7:e31041.

42. Cooper ST, McNeil PL. Membrane Repair: Mechanisms and Pathophysiology. Physiol Rev. 2015;95:1205-40.

43. Wang $X$, Tucker NR, Rizki G, Mills R, Krijger PHL, de Wit E, Subramanian V, Bartell $E$, Nguyen XX, Ye J, et al. Discovery and validation of sub-threshold genome-wide association study loci using epigenomic signatures. Elife. 2016;5:10557.

44. Hager HA, Roberts RJ, Cross EE, Proux-Gillardeaux V, Bader DM. Identification of a novel Bves function: regulation of vesicular transport. EMBO J. 2010;29: $532-45$.

45. Smith TK, Hager HA, Francis R, Kilkenny DM, Lo CW, Bader DM. Bves directly interacts with GEFT, and controls cell shape and movement through regulation of Rac1/Cdc42 activity. Proc Natl Acad Sci U S A. 2008;105:8298-303.

46. Benesh EC, Miller PM, Pfaltzgraff ER, Grega-Larson NE, Hager HA, Sung BH, Qu X, Baldwin HS, Weaver AM, Bader DM. Bves and NDRG4 regulate directional epicardial cell migration through autocrine extracellular matrix deposition. Mol Biol Cell. 2013;24:3496-510.

47. Parang B, Kaz AM, Barrett CW, Short SP, Ning W, Keating CE, Mittal MK, Naik $\mathrm{RD}$, Washington MK, Revetta FL, et al. BVES regulates c-Myc stability via PP2A and suppresses colitis-induced tumourigenesis. Gut. 2017;66:852-62.

48. Mamchaoui K, Trollet C, Bigot A, Negroni E, Chaouch S, Wolff A, Kandalla PK, Marie S, Di Santo J, St Guily JL, et al. Immortalized pathological human myoblasts: towards a universal tool for the study of neuromuscular disorders. Skelet Muscle. 2011;1:34.

49. Holt I, Fuller HR, Lam LT, Sewry CA, Shirran SL, Zhang Q, Shanahan CM, Morris GE. Nesprin-1-alpha2 associates with kinesin at myotube outer nuclear membranes, but is restricted to neuromuscular junction nuclei in adult muscle. Sci Rep. 2019;9:14202.

50. Nguyen TM, Cartwright AJ, Morris GE, Love D, Bloomfield JF, Davies KE. Monoclonal antibodies against defined regions of the muscular dystrophy protein, dystrophin. FEBS Lett. 1990;262:237-40.

\section{Publisher's Note}

Springer Nature remains neutral with regard to jurisdictional claims in published maps and institutional affiliations. 